\title{
Urological Surgery
}

National Cancer Institute

\section{Source}

National Cancer Institute. Urological Surgery. NCI Thesaurus. Code C105700.

A surgical specialty concerned with surgical treatment of the diseases of the urinary tract and urogenital system. 